\title{
UNCERTAINTY OF OUTCOME AND VARYING FAN PREFERENCES - A GAME THEORETIC APPROACH
}

\author{
KJETIL K. HAUGEN
}

\begin{abstract}
This paper applies simple game theory to investigate an equilibrium link between composition of football clubs' fans preferences and the clubs' talent acquisition decisions. Such a link is identified, and wealth of the clubs turns out to be important for such equilibria to be established. However, even poor clubs can reach equilibria where they end up being winners of the "talent-acquisition-game", given that their fans are 'die-hard' enough. In short; clubs with a long history and a dedicated fan base are much better prepared for successful competition in the football market.
\end{abstract}

\section{INTRODUCTION}

A 'die-hard' football fan might be defined as having a specially strong relationship to a particular team. Most of us who recognize such a definition, have perhaps been in match situations where our favourite team leads comfortably. In such situations, we experience that satisfaction is the most prominent feeling, and not an urge for a more even match. That is, we enjoy the situation of a secure win for our team. In a sports economic context such feelings may (at least to a certain extent) contradict the famous 'uncertainty-of-outcome-hypothesis' ${ }^{1}$ - see [13].

On the other hand, most of us also know, that if we watch football with less personal commitment to clubs or countries, we prefer even matches, even if we actually have a favourite among the teams playing.

Hence, the 'UoO-hypothesis' makes sense, but it may perhaps not be the imperative driver of fan preferences. The interesting thing in this situation, is that the two different preference-situations described above are contradictory. The 'die-hard' passionate fan loves a winning probability moving towards 1 , while the 'UoO-seeker' thrives for a winning probability closer to a half. Consequentially,

$M S C$ (2010): primary 91; secondary 91A10, 91A 80.

Keywords: game theory, uncertainty of outcome, different fan preferences, football (soccer).

Motivational support and inspiration from Prof. Tim Pawlowski is gratefully acknowledged. Tim contributed significantly in an early phase of this work. This research has received no special funding.

${ }^{1}$ The Uncertainty of outcome hypothesis, introduced in the fifties by Rottenberg, states that a competition, involving teams or individuals is only of interest for spectators if it contains uncertainty related to competition outcome. A certain win for any competitor makes watching the competition uninteresting for most spectators. On the other hand, too much uncertainty (i.e. an outcome probability close to a half) - a lottery - also makes the competition relatively uninteresting. After all, who wants to pay hard cash watching a coin being tossed. We refer to this hypothesis as the 'UoO-hypothesis' for the rest of this paper. 
if football audiences contain different spectators, (which they obviously do), such differences as described above should be present. And, more importantly, such preference differences may affect team equilibrium decisions; for instance related to talent acquisition.

In this paper, the above discussed problem is treated through simplified game theory. A game model framework, as presented in [7] is introduced and applied with some modifications. Section 2 is about the relevant literature. Section 3 presents the modelling framework and discusses the modification proposed. The actual game model and analysis is the subject of Section 4, followed by the final Section 5, which sums up, criticizes and concludes.

\section{Relevant Literature}

Literature on sport and fan motivation is rich, also within research. Sport sociology and sport psychology are concerned with these subjects - see, for instance $[4,16]$ or [9]. Much of these contributions are empirically based, often with information collected from questionnaires. A topic which has been especially well treated seems to be gender differences - see, e.g. [2] or [8]. However, this branch of literature has limited impact on the topic analysed here.

Sport marketing/media also (naturally) spend much resources on investigating different groups of consumers and possible differences in preferences - obviously motivated by strategies for exploiting various groups' potential differences in willingness to pay. However, again, most contributions are of minor relevance for this paper, where strategies and equilibria are in focus. Some examples of some (but limited) relevance may be found in [5,6] or [14].

Sport(s) Management/Economics both should be, and most certainly also are, the research literature segment of most relevance for this paper. Although even this literature segment is sparse (at most) of content of real relevance for the modelling approach used in this paper, there are some useful and interesting contributions which at least partially cover the relevant topics. The classical and rich discussion on the link between ' $\mathrm{UuO}$ ' and demand provides a necessary background (see, e.g. $[3,13,15])$ but is still limited to models involving various fan groups, and hence differing demand among such groups. Some recent work, although mainly empirical, by Pawlowski et. al. [10-12] does however raise relevant questions on whether different preferences among fans may support the 'UuO-hypothesis' or not.

\section{Game modelling CONSIDERATions}

As discussed in Section 1, I propose to apply a game model introduced in [7]. This game model is perhaps most efficiently explained by one of the figures in the paper.

The normal form game (of complete information) in Figure 1 contains two players. The two players (teams), named $T_{1}$ and $T_{2}$ choose actions from a common strategy space - $\left(E_{p}, C_{p}\right)$, where $E_{p}$ means buying expensive talent and $C_{p}$ means buying cheap talent. Consequently, a buying decision is assumed to be taken already, and the players of this game can either add talent providing increased 


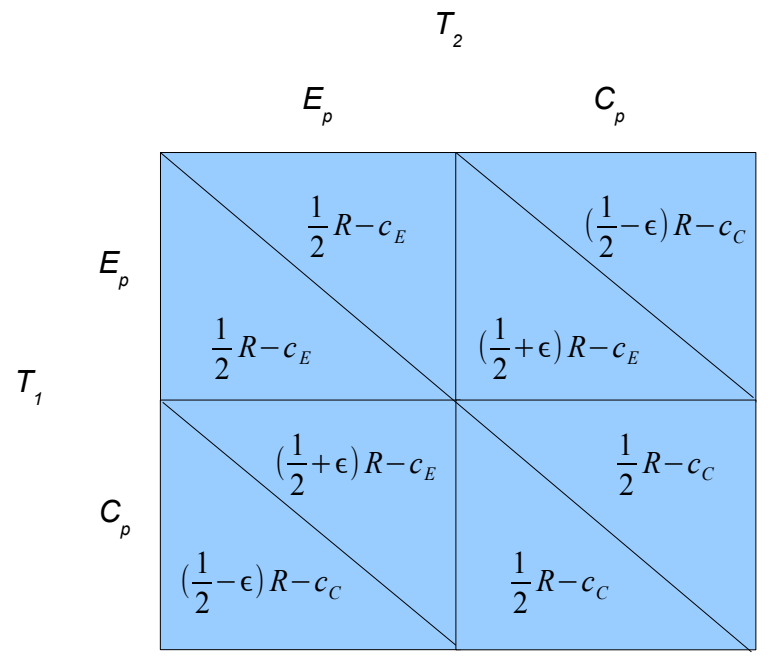

Figure 1. The Profit maximising single decisive match game (originally labelled Figure 3 in [7]).

or decreased probability of winning the given match. That is, the model assumes a single match of decisive nature, effectively ruling out a draw option. A simple way of thinking of the setting could be: Two teams deciding to reinforce their teams before of an upcoming match of great financial importance; say a champions League qualifier.

Teams are assumed cloned, and the same holds for players acquired of both types. As a consequence, if both teams buy the same talent $\left(E_{p}, E_{p}\right)$ or $\left(C_{p}, C_{p}\right)$, the probability of winning the match is $\frac{1}{2}$ for both teams, while some advantage is gained for one team if $E_{p}$ is chosen while the other team chooses $C_{p}$. This advantage is modelled by a single parameter $\epsilon$ in Figure 1. This $\epsilon$ is assumed to lead to an additive increase in match winning probability, and must hence logically lead to similar winning probability reduction if actions $\left(E_{p}, C_{p}\right)$ or $\left(C_{p}, E_{p}\right)$ are chosen by the teams. The prize of winning ${ }^{2}$ this match is named $R$, and is assumed to be the same for both teams in the basic model in Figure 1.

Now, the pay-off values in Figure 1 are easily recognized as expected pay-offs (or profits), given a definition of talent prices (costs) of $c_{E}$ and $c_{C}$ for expensive and cheap talent respectively.

There are two possible (unique) Nash equilibria (NEs) of this model ${ }^{3}$, both of the coordinating type; $\left\{E_{p}, E_{p}\right\}$ or $\left\{C_{p}, C_{p}\right\}$.

Now, in order to revise this model framework to fit the topic of this paper, we need to do a few things. As discussed previously, our main focus here is to investigate the effects of different consumer groups, with different attitudes to Uncertainty of Outcome. Without loss of generality game-theory-wise, we can restrict the number of fan-groups to two. We stick to the two groups loosely defined previously (Section 1). We label the 'die-hard' fans as type 1, and the

\footnotetext{
${ }^{2}$ Note that the assumed decisiveness of the game effectively rules out any draw option ${ }^{3}$ Depending on the direction of the inequality sign in $R \gtrless \frac{c_{E}-c_{C}}{\epsilon}$.
} 
'UoO-seeker' group as type 2. Then, we assume that both groups are present for both teams providing revenue generation for the match at hand. Consequently we can define:

$$
R_{i}=\alpha R_{i}^{1}+(1-\alpha) R_{i}^{2}
$$

where $\alpha$ defines the proportion of fans in each group. Note, that at this point, we open up for team specific revenue generation $\left(R_{i}\right)$ which seems obvious given the setting. The two clubs playing the match will in practice never have homogeneous fans.

To link this up with the uncertainty of outcome, it seems evident that a slightly more complex view of the revenue generation must be introduced. It is well known from Sports Economics theory (see, e.g. [1]) that demand, willingness to pay or realized willingness to pay (Revenue) is caused by many variables. Match-type, opponent, price, performance of teams and many other more or less influential variables are amongst such. In our setting, we simplify and focus on team performance (symbolized by winning percent $-W$ here) for each team. Figure 2 sums up this approach.

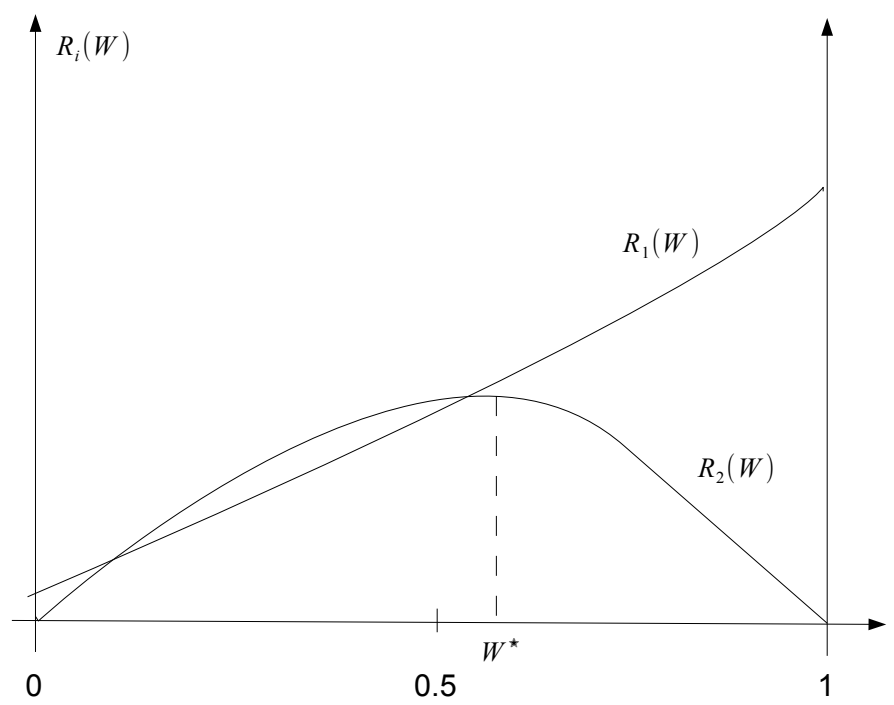

Figure 2. Exemplifying 'die-hard' $\left(R_{1}(W)\right)$ and 'UoO-seekers' $\left(R_{2}(W)\right)$ groups plotted as a function $W$.

Let us assume that each team has fans from one of the groups, but with opposite preferences. This corresponds (in equation (3.1)) to a situation where $\alpha=1$ if $i=1$ and $\alpha=0$ if $i=2$. Let us furthermore assume that the shapes of the $R$-functions are representative for the content we have hypothesized on these two groups. That is, group 1 contains 'die-hard' fans who prefer the highest possible probability of a home win, while group 2 - those who prefer an even game, would achieve maximal willingness to pay for some winning probability closer to a half. As Figure 2 indicates, the given curves correspond with such an assumption. 
More formally, we could state that

and

$$
\text { for } R_{1}: \frac{d R_{1}(W)}{d W}>0, \forall W \in[0,1]
$$

$$
\text { for } R_{2}:\left\{\begin{array}{ll}
\frac{d R_{2}(W)}{d W}>0 & \text { if } W \in\left[0, W^{*}\right] \\
\frac{d R_{2}(W)}{d W}<0 & \text { if } W \in\left[W^{*}, 1\right]
\end{array}\right. \text {. }
$$

Now, we are in a position to re-analyse the game of Figure 1. Observe that within this model framework, the only change implies substituting $R$ by $R_{1}$ and $R_{2}$ for a given level of winning percent.

\section{The (REvised) GAME MOdel AND NEs}

Figure 3 shows the redefined game as outlined in previous sections.

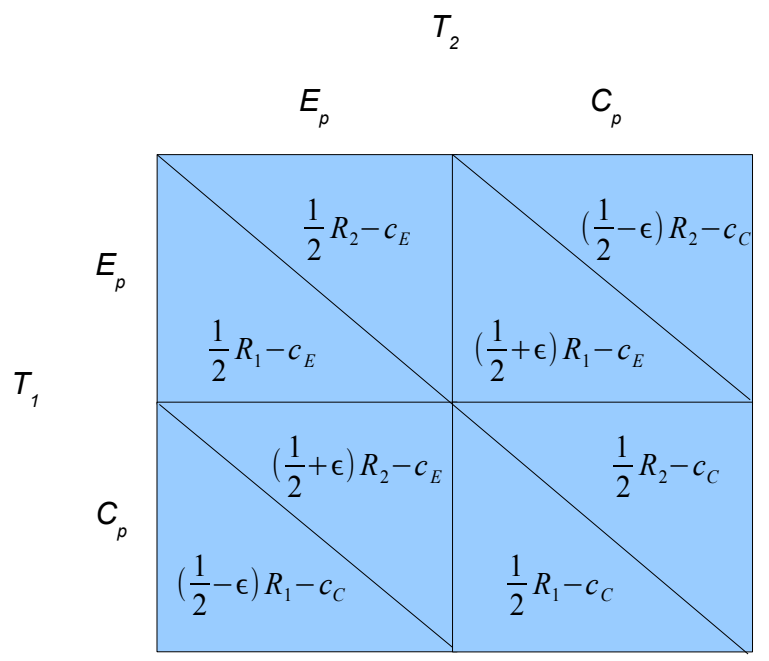

Figure 3. The revised game.

The game in Figure 3, is easily analysed. Perhaps not in general, but for our purpose, establishing the relevant new NEs turn out to be relatively straightforward. Surely, the coordinating NEs of the original game $\left(E_{p}, E_{p}\right)$ and $\left(C_{p}, C_{p}\right)$ are present and achievable, but what is interesting now, is that other types of NEs emerge. We will focus on pooling (or separating) NEs and start by identifying the necessary inequalities for existence of such. Let us start by looking at $\left\{E_{p}, C_{p}\right\}$ as a NE candidate. Then, the following best replies must be satisfied:

$$
\left(\frac{1}{2}-\epsilon\right) R_{2}-c_{C}>\frac{1}{2} R_{2}-c_{E}
$$

and

$$
\left(\frac{1}{2}+\epsilon\right) R_{1}-c_{E}>\frac{1}{2} R_{1}-c_{C}
$$


A tiny bit of algebra on equation (4.1) gives:

$$
\frac{1}{2} R_{2}-\epsilon R_{2}-c_{C}>\frac{1}{2} R_{2}-c_{E} \Rightarrow c_{E}-c_{C}>\epsilon R_{2} .
$$

A similar argument on equation (4.2) gives:

$$
\frac{1}{2} R_{1}+\epsilon R_{1}-c_{E}>\frac{1}{2} R_{1}-c_{C} \Rightarrow \epsilon R_{1}>c_{E}-c_{C} .
$$

If we combine equations (4.3) and (4.4), we get:

$$
\epsilon R_{1}>c_{E}-c_{C}>\epsilon R_{2}
$$

and finally reducing by $\epsilon$ in equation (4.5)

$$
R_{1}>\frac{c_{E}-c_{C}}{\epsilon}>R_{2} .
$$

If we perform an analysis focusing on the other interesting $\mathrm{NE}-\left\{C_{p}, E_{p}\right\}$. We find inequalities similar to (4.1) and (4.2):

$$
\begin{aligned}
& \left(\frac{1}{2}-\epsilon\right) R_{1}-c_{C}>\frac{1}{2} R_{1}-c_{E}, \\
& \left(\frac{1}{2}+\epsilon\right) R_{2}-c_{E}>\frac{1}{2} R_{2}-c_{C} .
\end{aligned}
$$

Proceeding as above, we find:

$$
R_{2}>\frac{c_{E}-c_{C}}{\epsilon}>R_{1} .
$$

If we compare inequalities (4.6) and (4.7), it is readily observed that they cannot be both satisfied simultaneously. From a game theoretic point of view, this is relevant, as it means that the "Chicken-type" NE-situation can not be obtained. That is, a situation where both the $\left\{C_{p}, E_{p}\right\}$ and the $\left\{E_{p}, C_{p}\right\}$ NEs (accompanied by a third NE in mixed strategies) is not a legal outcome of the game. As a consequence, either a pure $\left\{C_{p}, E_{p}\right\}$ or a pure $\left\{E_{p}, C_{p}\right\} \mathrm{NE}$ will be realized. This is an interesting, and by no means obvious conclusion. The fact that the situation at hand completely excludes the possibility of multiple NEs signals predictable game outcomes.

Let us now return to the assumptions underlying Figure 2 and the then relevant inequality - (4.6). An obvious conclusion to draw then, is that if this inequality is satisfied, the only game prediction we observe is Team 1 buying expensive while team 2 buys cheap. In layman terms, and according to the model predictions, Team 1 who has only 'die-hard' fans enforces their team, while Team 2 (with only thrill-seeking supporters) chooses to buy cheap and hence downgrade their team quality relative to Team 1 . We view this as an interesting conclusion. We have demonstrated that differing fan preferences directly impact team equilibrium decisions related to talent acquisition. In a more long-term perspective, many decisive matches should then also impact competitive balance. The teams with only (or a majority of) 'die-hard' supporters are (in this sense) the winners. A team such as Chelsea (who seemingly has changed their fans) might hence be a nine days' wonder compared to other PL-teams like Liverpool or MUFC. To some extent, it might even be possible to do some empirical testing of this type of conclusion. 
Surely, for such a conclusion to make any real-world sense, inequality (4.6) is vital. More specifically, when will it be satisfied, and are we able to give any general statements on whether reality could be expected to produce a valid inequality (4.6)? Obviously, at least we need to be in a setting where teams have different revenue functions. That is, some teams must generate more revenue than others. However, inequality (4.6) does not only demand that $R_{1}>R_{2}$, it must also be bigger by the amount of $\frac{c_{E}-c_{C}}{\epsilon}$. Alternatively, this fraction may be very small, either if $c_{E} \approx c_{C}$ or if $\epsilon$ is big or both. Hence, in a practical setting, if the "rich" teams $\left(R_{1}\right)$ are able to buy talent relatively cheap compared to the "poor" teams, or they are able to get significantly better players (even at a high price) than their competitors, $\frac{c_{E}-c_{C}}{\epsilon}$ will be small and it is in a sense enough to be richer than the competing club to expect this NE to emerge. But, and this is important, a club does not need to be richer than another for this NE to be realized. Take a look at Figure 4.

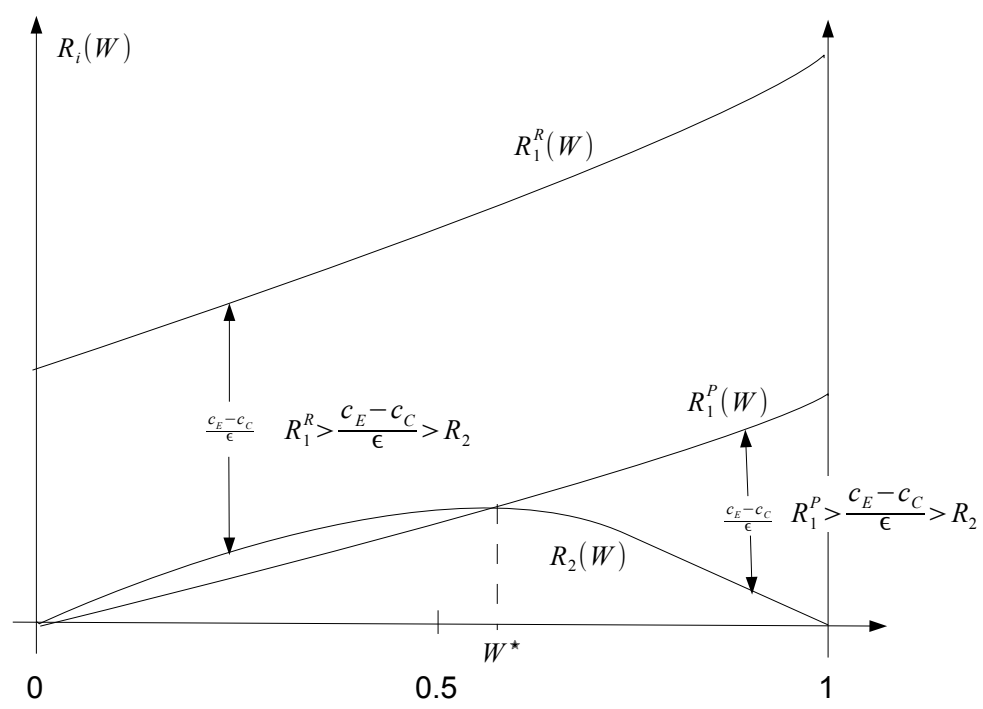

Figure 4. Different revenue functions for team 1: a very rich vs. a less rich team.

Figure 4 shows two different versions of Team 1's revenue function: $R_{1}^{P}(W)$ and $R_{1}^{R}(W)$, as well as Team 2's revenue function: $R_{2}(W)$. As can be observed from Figure 4, the rich team $\left(R_{1}^{R}(W)\right)$ will provide a satisfied inequality (4.6) for any $W$. Obviously, this is correct for the (geometrical) choice of value for $\frac{c_{e}-c_{c}}{\epsilon}$ as shown in the figure. Likewise (obviously), if $\frac{c_{e}-c_{c}}{\epsilon}$ increases such that (4.6) no longer holds, we can obtain satisfaction for (4.6) by simply lifting $R_{1}^{R}(W)$. As a consequence, the separating $\mathrm{NE} ;\left\{E_{p}, C_{p}\right\}$, is very likely to occur. However, and more importantly, by the actual winning percent $W$, a poorer club (in a more balanced league) can also (if a high winning percent is achieved) reach the same 
type of NE. This is of course all very well and intuitive. A very reach team will buy the most expensive players, leaving the less expensive (and expectantly of less quality) players to its competitors. This effect can be observed at the bottom in Figure 4, where team 1 may be viewed as 'poorer' than a team for $W$ 's lower than $W^{*}$. Also here, higher values for $\frac{c_{e}-c_{c}}{\epsilon}$ can be 'compensated' by lifting $R_{1}^{P}(W)$ in the area close to $W=1$.

The really interesting thing here is that the fan-base itself, given adequately different, could drive a similar NE. As long as the fans are "enough" interested in their team winning, a team that is "poorer" for most $W$ s can still end up with inequality (4.6) satisfied, and hence acquiring the best and most expensive players.

Another interesting consequence of this analysis is the fact that the composition of the fan base for teams actually influence competitive balance in leagues. If one would like to avoid these types of effects completely, one would have to make all clubs fans equal. A strikingly unpleasant scenario by the way. On the other hand, our results show how complex football, its fans and the football economy actually is.

In the real world, no clubs have either fans of type 1 or 2 . All real-world clubs will have some of each category, and perhaps even of other additional categories. Still, our arguments will hold if the share of 'die-hard' fans is high enough.

\section{Conslusions And SUgGestions FOR FURTher WORK}

The fact that football teams with dedicated supporters have a a better survival equilibrium property than teams with less dedicated supporters may perhaps be observed as the main conclusion of this paper. Surely, this should not come as much of a surprise to football practitioners. On the other hand, when hooliganism and trouble pops up, such information may be good to recall.

The fact that wealth provides such an equilibrium outcome more easily is possibly not very surprising either. However, the fact that even poor clubs, with fans dedicated enough, can overcome richer clubs is in my opinion a less expected conclusion. Obviously, such an outcome is harder to achieve, but it is a nice feature of inherent "Robin-Hood-ness" in the football market.

To a certain extent, we could possibly state this as follows: If you are a small and poor football club, as long as you are able to provide supporters with enough dedication (significantly more dedicated than your competitors), you will (in the long run) be able to compete and improve; reaching the top of the table. This ought to be good news for teams and countries, dreaming about beating Barcelona or Brazil.

Unfortunately, these easy and reassuring conclusions are derived from a model based on several grave simplifications of reality. Apart from the fact that we examine a single game between only two teams ${ }^{4}$, the model exogeneity of fan preferences is obviously an important and perhaps too harsh simplification. Recall that our model set-up takes fan preferences, by the revenue functions $R_{1}^{P}, R_{1}^{R}, R_{2}$ as given. Obviously, fan preference structures (e.g. being dedicated) are formed

\footnotetext{
${ }^{4}$ Personally, I do not believe this to be the primal questionable assumption. After all, if one should put any faith to the football industry's talk about "Taking one match at the time", the league as such may perhaps not play such an important role.
} 
by a much more complex dynamic game. That is, teams' decisions related to team performance affect their fans and how dedicated fans are and will be in the future. This (obvious) feedback is clearly not a part of the modelling of this paper. Still, in a shorter time perspective, I feel that the conclusions hold both scientific merit as well as practical usability.

Surely, the conclusions above should be (at least in principle) empirically testable. Reality will of course not provide teams with either 'die-hard fans' or 'UuO-seekers', most teams will have supporters of both types, and perhaps even of other types as well. Still, as argued above, if one is able to find clubs with especially dedicated fans and compare such a group of clubs with other clubs with less dedicated fans (they definitely exist) one could observe reality and see if any of the conclusions above are observable. Such a task is by no means simple, but possible and interesting as an addition to the pure modelling approach presented here.

\section{REFERENCES}

[1] J. Borland, Demand for sport, Oxford Rev. Econ. Policy 19 (2003), 478-502.

[2] S. T. Eastman and A.C. Billings, Biased voices of sports: Racial and gender stereotyping in college basketball announcing, Howard J. Commun. 12 (2001), 183-201.

[3] M. El-Hodiri and J. Quirk, An economic model of a professional sports league, J. Polit. Econ. 79 (1971), 1302-1319.

[4] D.C. Funk and J. James, The psychological continuum model: A conceptual framework for understanding an individual's psychological connection to sport, Sport Manag. Rev. 4 (2001), 119-150.

[5] D. C. Funk, D. F. Mahony and L. L. Ridinger, Characterizing consumer motivation as individual difference factors: Augmenting the sport interest inventory (sii) to explain level of spectator support, Sport Mark. Q. 11 (2002), 33-43.

[6] K. Gwinner and S. R. Swanson, A model of fan identification: Antecedents and sponsorship outcomes, J. Services Marketing 17 (2003), 275-294.

[7] K. K. Haugen and H. A. Solberg, The financial crisis in European football - a game theoretic approach, Eur. Sport Manag Q. 10 (2010), 553-567.

[8] J. D. Jeffrey and L. L. Ridinger, Female and male sport fans: A comparison of sport consumption motives, J. Sport Behav. 25 (2002), 260-278.

[9] R. Madrigal. Cognitive and affective determinants of fan satisfaction with sporting event attendance, J. Leisure Res. 27 (1995), 205-227.

[10] G. Nalbantis, T. Pawlowski and D. Coates, The fans' perception of competitive balance and its impact on willingness-to-pay for a single game, J. Sports Econ., published online before print, May 26, 2015.

[11] T. Pawlowski, Testing the uncertainty of outcome hypothesis in European professional football: A stated preference approach, J. Sports Econ. 14 (2013), 341-367.

[12] T. Pawlowski, Wettbewerbsintensität im Profifußball, Springer-Gabler Research, Wiesbaden, 2013.

[13] S. Rottenberg, The baseball player's labor market, J. Polit. Econ. 64 (1956), 242-258.

[14] R. L. Snipes and R. Ingram, Motivators of collegiate sport attendance: A comparison across demographic groups, Innovative Marketing 3 (2007), 65-74.

[15] S. Szymanski, Income inequality, competitive balance and the attractiveness of team sports: Some evidence and a natural experiment from english soccer, Econ. J. 111 (2001), 69-84.

[16] D. L. Wann, M.P. Schrader and A. M. Wilson, Sport fan motivation: Questionnarie validation, comparisons by sport, and relationship to athletic motivation, J. Sport Behav. 22 (1999), 114-139. 
Kjetil K. Haugen, Faculty of Logistics, Molde University College, Specialized University in Logistics, Britvegen 2, 6410 Molde, Norway

e-mail: kjetil.haugen@himolde.no 